\title{
Mathematical Simulation and Solution of the Problem of Seismo-Dynamics of Underground Pipelines
}

\author{
D.A. Bekmirzaev ${ }^{1}$, R.U. Kishanov ${ }^{2}$, N.Sh. Mansurova ${ }^{3}$ \\ ${ }^{1}$ Institute of Mechanics and Seismic Stability of Structures, Tashkent, Uzbekistan, diyorbek_84@mail.ru \\ ${ }^{2}$ Tashkent State Transport University, Tashkent, Uzbekistan, ruslan_k@mail.ru \\ ${ }^{3}$ Namangan Engineering Construction Institute, Namangan, Uzbekistan, mansurovan@mail.ru
}

\begin{abstract}
Mathematical modeling and numerical solution of problems of underground pipelines under spatial seismic effects are discussed in the paper. The systems of differential equations of motion are obtained for the problems of seismodynamics of underground pipelines with complex conditions of interacting with soil, at arbitrary direction of seismic load, based on Hamilton-Ostrogradsky variation principle, this allows in general form to consider a number of classes of problems. An analysis of results was made for an extended pipeline with joints located on the $O x y$ plane and interacting with surrounding soil. Applied software was created for calculation and visual representation of the results of solving problems in the form of graphs of changes in displacements, forces and moments over time at selected nodal points of the structure and in the form of animation. Results obtained present a new contribution into seismodynamic theory; they open wide possibilities for their use in optimal design of the complex of life support in seismic zones.
\end{abstract}

Key words: model, underground pipeline, seismic wave, interaction, soil, numerical method.

\section{INTRODUCTION}

At present, the case-history of strong earthquakes that have occurred in the world shows that a preliminary hazard assessment of earthquakes and timely consideration of appropriate measures (problems of assessing seismic risk and their mitigation) are of great importance for the reduction of catastrophic aftermath. Thus, it is necessary to ensure the strength and stability of underground structures subjected to seismic loads. In this sense, the implementation of targeted scientific research to improve numerical methods for solving problems, to calculate the earthquake resistance of underground pipeline systems under random seismic effects is a relevant issue [1-3].

The current capability of computing facilities makes it possible to more fully account for numerous factors and, with a greater degree of reliability, determine the actual stress-strain state of an underground pipeline.

Underground pipelines serve as a primary part of the life-support system of cities and centers of population (water-, gas- and heat supply, sewer system), objects of extraction and transportation of oil and gas; their safe operation especially in seismic zones is very important [1-14].

Severe earthquakes all over the world in recent years (Nepal, 15-26 April, 2015; Chile, 2 April, 2014; Cephalonia, 26 January and 3 February, 2014; Japan, 13 April, 2013 and others) affected normal operation of underground support networks; their number growing considerably high in 1960-1980 years of the last century due to urbanization of towns and villages.

In the second half of the twentieth century to calculate the complex system of underground pipelines with a large number of branches and inclusions (water supply, drainage systems, etc.) T.R. Rashidov has proposed "Dynamic theory of seismic stability of complex systems of underground structures", based on the presentation of linear part of the pipeline by core elements of finite length and joint connections (structural inclusion) - by absolutely rigid bodies.

At present abroad there have already appeared several scientific research works, which state not only the problems of seismic stability of underground structures, but also the formation of normative documents on their calculation and design (Japan, USA, China, Russia and others). This is facilitated, on the one hand, by active development of urban areas, expansion of support system networks, on the other hand, by sufficient number of strong earthquakes in the world, which caused damage to underground networks.

Tashkent earthquake (1966) has also caused serious damage and destruction to underground networks of life support - to water supply pipelines, sewer systems and other. However, as noted in the paper, this was observed only in cases when the pipes were laid in soil of a certain bearing capacity.

In works of S. Sarioletlagh, M. Nekooei, A. Aziminejad, D. Ha, T.H. Abdoun, M.J. O'Rourke, M.D. Symans, M. Saberi, H. Arabzadeh, A. Keshavarz, P. Vazouras, S.A. Karamanos, et al. the use of different mechanical mathematical models was analyzed and a number of urgent problems of underground and ground structures were solved [4-14].

The results of theoretical and practical research in the field of seismic stability of underground pipelines were analyzed in 
detail in monographs by M.J. O'Rourke and X.L. Liu (1999). A deep analysis of the results of this monographic study allows us to trace the history of formation and development of seismodynamics worldwide. There were only a few data on the consequences of the earthquake in Japan (Tokyo, 1923), United States (California, 1906), Turkmenistan (Ashgabat), Uzbekistan (Tashkent), and others. This is explained by the fact that the length of underground pipelines in seismically active areas was comparatively short and it was difficult to find the damage.

This paper is devoted to the study of the underground pipelines dynamics based on the seismodynamic theory of underground structures, using a mathematical model of the theory of rods, considered in [1-2] for the case of rod point displacements under combined action of longitudinal and transverse forces.

\section{MATHEMATICAL SIMULATION DYNAMICS OF UNDERGROUND PIPELINES}

In seismodynamic theory of underground structures the studies on the problem of interaction in the «structure-soil» system have a primary importance. In this connection, various laws of soil-structure interaction are developed; they consider the parameters characterizing the process of contact interaction of deformable rigid bodies with soil [13].

Pipeline life support systems consist of straight-line sections connected by joints orthogonally and non-orthogonally coupled together. A seismic wave initiated during an earthquake affects such a system of pipelines at an arbitrary angle of attack in space. For an underground system of arbitrarily located pipelines with an arbitrary angle of attack of seismic effect in space, it is necessary to develop new computational mathematical models and software for determining the stress-strain state [3].

To study the longitudinal, torsional and transverse vibrations of underground pipelines under randomly directed seismic load, the applied theory of rod vibrations was used [9-11].

The Hamilton-Ostrogradsky variational principle for an underground pipeline has the form [1-2]

$\int_{t}(\delta \mathrm{T}-\delta \Pi+\delta \mathrm{A}) d t=0$,

where $\delta \mathrm{T}, \delta \Pi$ are the variations of kinetic and potential energies; $\delta \mathrm{A}$ is the variation of the work of external forces; $\mathrm{t}$ is time.

The pipeline is modeled as a rod. Displacements are defined as follows [1-2]:

$u_{1}=u-y \alpha_{1}-z \alpha_{2}, u_{2}=\mathrm{v}+z \theta, u_{3}=w-y \theta$,

where the components $u_{1}, u_{2}, u_{3}$ are the displacements of any point in the pipeline; $u, \mathrm{v}, w-$ are the longitudinal, transverse horizontal and vertical displacements of the pipeline axis; $\alpha_{1}, \alpha_{2}$ are the angles of rotation of the pipeline axis under pure bending; $\theta$ is the torsion of the pipeline.
Variations in kinetic energy of the pipeline. Kinetic energy variations can be represented as

$$
\begin{aligned}
& \int_{t} \delta \mathrm{T} d t=\iint_{t}\left[\rho \frac{\partial u_{1}}{\partial t} \delta \frac{\partial u_{1}}{\partial t}+\right. \\
& \left.+\rho \frac{\partial u_{2}}{\partial t} \delta \frac{\partial u_{2}}{\partial t}+\rho \frac{\partial u_{3}}{\partial t} \delta \frac{\partial u_{3}}{\partial t}\right] d V d t .
\end{aligned}
$$

Variations in potential energy of the pipeline. Consider the potential energy variations

$$
\int_{t} \delta \Pi d t=\int_{t} \int_{V}\left(\sigma_{11} \delta \varepsilon_{11}+\sigma_{12} \delta \varepsilon_{12}+\sigma_{13} \delta \varepsilon_{13}\right) d V d t .
$$

Metal pipelines are strained within the elastic limit. Therefore, for a metal pipeline the Hooke's law is considered

$$
\begin{aligned}
& \sigma_{11}=E\left(\frac{\partial u}{\partial x}-y \frac{\partial \alpha_{1}}{\partial x}-z \frac{\partial \alpha_{2}}{\partial x}\right) \\
& \sigma_{12}=G\left(\frac{\partial u_{2}}{\partial x}+\frac{\partial u_{1}}{\partial y}\right)=G\left(\frac{\partial \mathrm{v}}{\partial x}+z \frac{\partial \theta}{\partial x}-\alpha_{1}\right), \\
& \sigma_{13}=G\left(\frac{\partial u_{3}}{\partial x}+\frac{\partial u_{1}}{\partial z}\right)=G\left(\frac{\partial w}{\partial x}-y \frac{\partial \theta}{\partial x}-\alpha_{2}\right) .
\end{aligned}
$$

For underground polymer pipelines, the stress-strain relation is:

$$
\begin{aligned}
& \sigma_{11}=E\left(\frac{\partial u}{\partial x}-y \frac{\partial \alpha_{1}}{\partial x}-z \frac{\partial \alpha_{2}}{\partial x}\right)\left(1-\int_{0}^{t} \Gamma(t-\tau) d \tau\right), \\
& \sigma_{12}=G\left(\frac{\partial \mathrm{v}}{\partial x}+z \frac{\partial \theta}{\partial x}-\alpha_{1}\right)\left(1-\int_{0}^{t} \Gamma(t-\tau) d \tau\right), \\
& \sigma_{13}=G\left(\frac{\partial w}{\partial x}+y \frac{\partial \theta}{\partial x}-\alpha_{2}\right)\left(1-\int_{0}^{t} \Gamma(t-\tau) d \tau\right) .
\end{aligned}
$$

Based on stresses $\sigma_{i j}$ (5) and (6), the longitudinal and transverse forces $N_{x}$ and $Q_{\mathrm{i} j}$, and the moments of bending and torques $M_{i j}$ were calculated.

Variations in external forces work of the pipeline. Variations in external forces work of the pipeline in a general way are as follows

$$
\begin{aligned}
& \int_{t} \delta A d t=\int_{t} \int_{V}\left[P_{1} \delta u_{1}+P_{2} \delta u_{2}+P_{3} \delta u_{3}\right] d V d t+ \\
& +\int_{t} \int_{S}\left[q_{1} \delta u_{1}+q_{2} \delta u_{2}+q_{3} \delta u_{3}\right] d S d t+ \\
& +\left.\int_{t} \int_{S_{1}}\left[\varphi_{1} \delta u_{1}+\varphi_{2} \delta u_{2}+\varphi_{3} \delta u_{3}\right] d S_{1} d t\right|_{x} .
\end{aligned}
$$

Here $P_{1}, P_{2}, P_{3}$ are the volume forces, $q_{1}, q_{2}, q_{3}$ are the surface forces, $\varphi_{1}, \varphi_{2}, \varphi_{3}$ are the lateral forces acting on the pipeline.

After setting the variation of kinetic (3), potential (4) energy and the work of external forces (7) based on the HamiltonOstrogradsky variational principle (1) and performing the operation of similar terms reduction, we obtain the variational equation of underground pipelines with the corresponding initial and boundary conditions. 


$$
\begin{aligned}
& \int_{t}(\delta \mathrm{T}-\delta \Pi+\delta \mathrm{A}) d t=\int_{x}\left[\rho \left(F \frac{\partial u}{\partial t} \delta u+I_{z} \frac{\partial \alpha_{1}}{\partial t} \delta \alpha_{1}+\right.\right. \\
& \left.\left.+I_{y} \frac{\partial \alpha_{2}}{\partial t} \delta \alpha_{2}+F \frac{\partial \mathrm{v}}{\partial t} \delta \mathrm{v}+F \frac{\partial w}{\partial t} \delta w+I_{p} \frac{\partial \theta}{\partial t} \delta \theta\right)\right]\left.d x\right|_{t}+ \\
& +\int_{t} \int_{x}\left\{\left[-\rho F \frac{\partial^{2} u}{\partial t^{2}}+\frac{\partial N_{x}}{\partial x}+N_{x}\left(P_{1}\right)+N_{x}\left(q_{1}\right)\right] \delta u+\right. \\
& +\left[-\rho I_{z} \frac{\partial^{2} \alpha_{1}}{\partial t^{2}}-\frac{\partial M_{z}}{\partial x}+Q_{12}-M_{z}\left(P_{1}\right)-M_{z}\left(q_{1}\right)\right] \delta \alpha_{1}+ \\
& +\left[-\rho I_{y} \frac{\partial^{2} \alpha_{2}}{\partial t^{2}}-\frac{\partial M_{y}}{\partial x}+Q_{13}-M_{y}\left(P_{1}\right)-M_{y}\left(q_{1}\right)\right] \delta \alpha_{2}+ \\
& \left.+\left[-\rho F \frac{\partial^{2} v}{\partial t^{2}}+\frac{\partial Q_{12}}{\partial x}+Q_{12}\left(P_{2}\right)+Q_{12}\left(q_{2}\right)\right)\right] \delta \mathrm{v}+ \\
& +\left[-\rho F \frac{\partial^{2} w}{\partial t^{2}}+\frac{\partial Q_{13}}{\partial x}+Q_{13}\left(P_{3}\right)+Q_{13}\left(q_{3}\right)\right] \delta w+ \\
& \left.+\left[-\rho I_{p} \frac{\partial^{2} \theta}{\partial t^{2}}+\frac{\partial M_{x}}{\partial x}+M_{x}\left(P_{2}, P_{3}\right)+M_{x}\left(q_{2}, q_{3}\right)\right] \delta \theta\right\} d x d t+ \\
& +\left\{\left[\left(-N_{x}+N_{x}\left(\varphi_{1}\right)\right) \delta u+\left(M_{z}-M_{z}\left(\varphi_{1}\right)\right) \delta \alpha_{1}+\right.\right. \\
& +\left(M_{y}-M_{y}\left(\varphi_{1}\right)\right) \delta \alpha_{2}+\left(-Q_{12}+Q_{12}\left(\varphi_{2}\right)\right) \delta \mathrm{v}+ \\
& \left.\left.\left.+\left(-Q_{13}+Q_{13}\left(\varphi_{3}\right)\right) \delta w+\left(-M_{x}+M_{x}\left(\varphi_{2}, \varphi_{3}\right)\right) \delta \theta\right]\right]_{x}\right\} d t=0 .
\end{aligned}
$$

where

$$
\begin{aligned}
& N_{x}=E F \frac{\partial u}{\partial x}, M_{z}=-E I_{z} \frac{\partial \alpha_{1}}{\partial x}, \\
& M_{y}=-E I_{y} \frac{\partial \alpha_{2}}{\partial x}, Q_{12}=G F \frac{\partial \mathrm{v}}{\partial x}-G F \alpha_{1}, \\
& Q_{13}=G F \frac{\partial w}{\partial x}-G F \alpha_{2}, M_{x}=G I_{p} \frac{\partial \theta}{\partial x} .
\end{aligned}
$$

Based on this statement, a system of differential equations, natural boundary and initial conditions is obtained from the variational equation. The resulting systems of differential equations and natural boundary and initial conditions can be represented in a vector form.

The system of differential equations for linear sections of underground pipelines in a local (for each linear section) spatial coordinate system (Oxyz) under random seismic action, with corresponding boundary conditions at the ends and initial conditions, has a vector form

$$
\begin{aligned}
& M \frac{\partial^{2} U}{\partial t^{2}}+A \frac{\partial^{2} U}{\partial x^{2}}+B \frac{\partial U}{\partial x}+C U=C U_{0}, \\
& D \frac{\partial U}{\partial x}+Q U=Q U_{0}, \\
& U=0, \quad \frac{\partial U}{\partial t}=0, \text { at } t=0 .
\end{aligned}
$$

where $M, A, B, C, D, Q$ are the sixth-order matrices, $U$ are the pipeline and nodes displacements, $U_{0}$ are the given displacements of soil under the effect of seismic waves, depending on time and coordinates.
The stresses in the underground pipeline generated from axial force $N$ and the combined action of axial force and the force moment $M$ under random action are calculated according to the following formulas

$$
\begin{aligned}
& \sigma=\frac{N}{F}, \\
& \sigma_{y, z}^{ \pm}=\frac{N}{F} \pm \frac{M_{y, z} \cdot R_{H}}{I_{y, z}} .
\end{aligned}
$$

The finite element method (FEM) in spatial coordinates and the implicit finite difference method (FDM) in time to discretize the pipeline problem under the action of random seismic waves are used as numerical methods for solving the equation of motion (9) with account for (10) and (11).

In this paper, an approach is proposed for determining the stress-strain state of a pipeline subjected to randomly directed plane longitudinal unsteady-state seismic wave. Its propagation direction makes an angle $\alpha$ with the pipeline axis $O x$ and $\beta$ is the angle between the projection of this wave direction vector onto the $O y z$ plane and the $O y$ axis of the pipeline.

Consider an extended pipeline with joints located on the Oxy plane and interacting with surrounding soil according to a simplified elastic model. Let the length of underground pipeline be $200 \mathrm{~m}$ and the length of each section $-5 \mathrm{~m}$ (Figure 1).

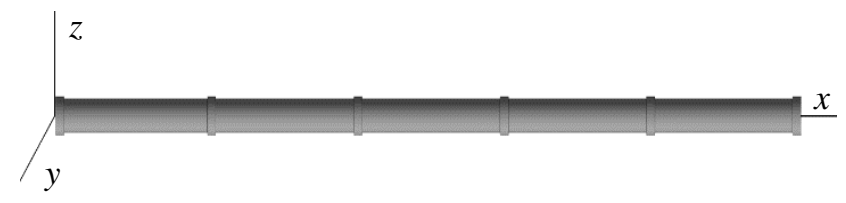

Figure 1: Underground pipeline with joint

The left and right ends of the pipeline are embedded into the ground, and the seismic wave is given as a harmonic function with incidence angles $\alpha=30^{\circ}, \beta=30^{\circ}$. In general, the problem is an unsteady-state spatial one for studying the processes in underground pipelines under the action of seismic waves.

Mechanical and geometrical parameters of underground pipeline and soil are taken as follows:

Problem. $\quad E=1.2 \cdot 10^{5} \mathrm{MPa} ; \quad \rho=7.0 \cdot 10^{3} \mathrm{~kg} / \mathrm{m}^{3} ; \quad l=200 \mathrm{~m}$; $D_{H}=0.5 \mathrm{~m} ; \quad D_{B}=0.49 \mathrm{~m} ; \quad$ in a straight section $k_{x}=1.5 \cdot 10^{4} \mathrm{kN} / \mathrm{m}^{3} ; k_{y, z}=3.9 \cdot 10^{4} \mathrm{kN} / \mathrm{m}^{3} ; v_{\text {soil }}=0.2 ; \quad v_{\text {pipe }}=0.3$; $u_{0}=a_{0} \cdot \sin \omega\left(t-x \cdot \cos \alpha / C_{p}\right) \cdot H\left(t-x \cdot \cos \alpha / C_{p}\right) ; \quad a_{0}=0.01 \mathrm{~m} ;$ $\omega=2 \pi / T ; \quad T=0.3 \mathrm{~s} ; \quad C_{p}=700 \mathrm{~m} / \mathrm{s} ; \quad u_{0 x}=u_{0} \cdot \cos \alpha$; $u_{0 y}=u_{0} \cdot \sin \alpha \cdot \cos \beta ; u_{0 z}=u_{0} \cdot \sin \alpha \cdot \sin \beta$.

The amplitude of ground vibrations $a_{0}$ corresponds to a 9 point earthquake on the MSK-64 scale. Joint stiffness factors are

$$
\begin{gathered}
K_{N}=80 \cdot 10^{4} \mathrm{kN} / \mathrm{m} ; K_{Q}=200 \cdot 10^{4} \mathrm{kN} / \mathrm{m} ; \\
K_{M_{u}}=9 \cdot 10^{2} \mathrm{kNm} ; K_{M_{k}}=8 \cdot 10^{4} \mathrm{kNm} .
\end{gathered}
$$

Here $E, D_{H}$ are the elastic modulus of the pipeline material and the outer diameter of the pipe, respectively; $\rho$ is the density of the pipeline material; $k_{x}, k_{y, z}$ are the coefficients of longitudinal, transverse and vertical interaction of the pipe 
with soil; $a_{0}$ is the amplitude of ground vibration; $\omega$ is the vibration frequency; $C_{p}$ is the propagation velocity of seismic waves in soil.

\section{RESULTS AND DISCUSSIONS}

Let's analyse the results. Figure 2 shows the changes in longitudinal displacements along the pipeline axis at a fixed time. The wave distribution along the pipeline length in the time range $t=0.05 \div 0.3 \mathrm{~s}$ is presented here. It was found that at $t=0.286 \mathrm{~s}$ the wave propagating in soil reaches the right end of the pipeline (Figure 2).

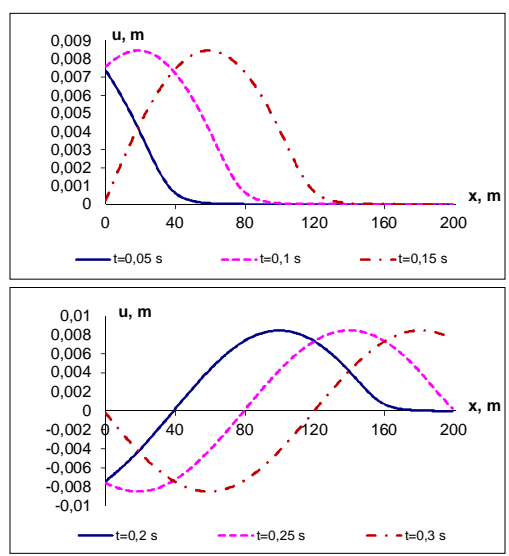

Figure 2: Changes in longitudinal displacements along the pipeline axis at a fixed time

Figure 3 shows the changes in transverse and vertical displacements along the pipeline axis at a fixed time.

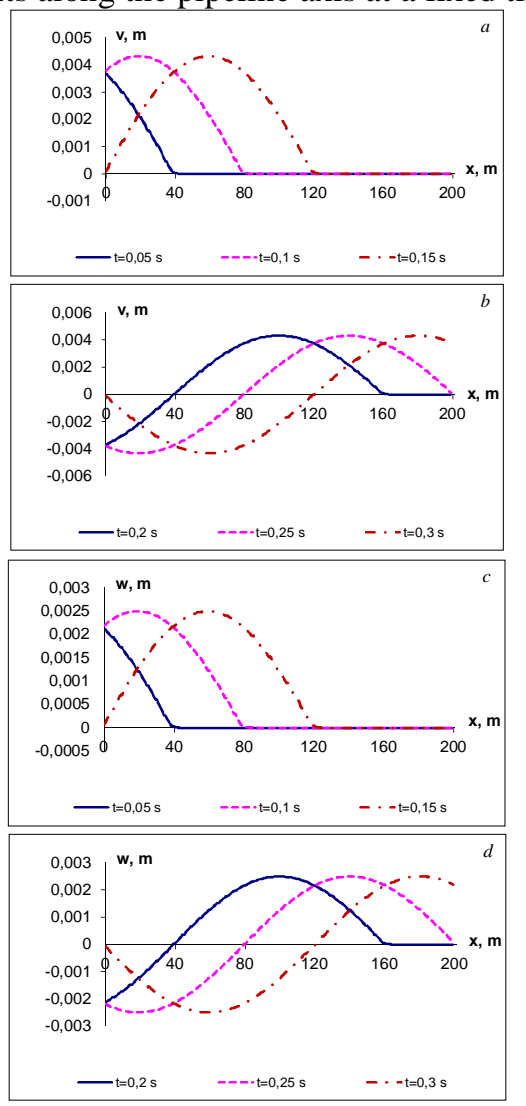

Figure 3: Changes in transverse $(a, b)$ and vertical $(c, d)$ displacements along the pipeline axis at a fixed time
The results show that the displacements in each section of the underground pipeline along the coordinate are complex in nature with certain small bursts associated with the presence of flexible joints.

Figure 4 shows the changes in compressive (tensile) stress along the axis of underground pipeline at a fixed time. The results show that in each joint, the compressive (tensile) stress of underground pipeline along the coordinate has a value distinctive for this joint with certain small bursts. This is due to the wave reflection from the joint and the wave propagation through the joint to the adjacent section of the pipeline.

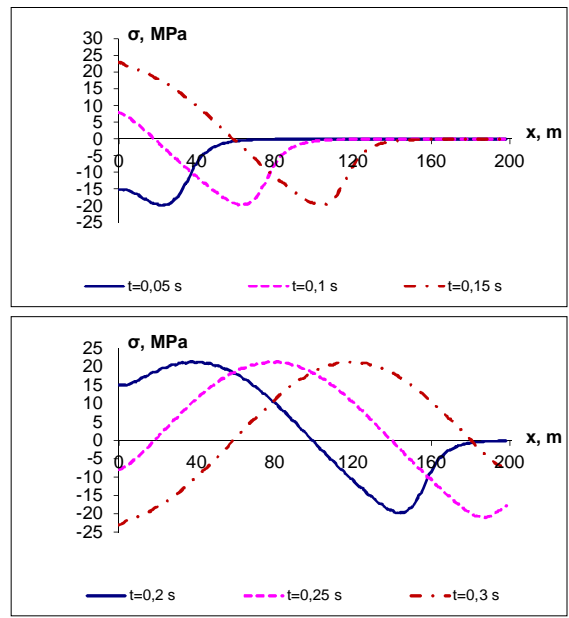

Figure 4: Changes in compressive (tensile) stress along the axis of underground pipeline at a fixed time

Figure 5 shows the changes in total stresses $\left(\sigma_{\mathrm{y}}{ }^{+}, \sigma_{\mathrm{y}}{ }^{-}\right)$along the axis of the underground pipeline at a fixed time. It is seen that at each joint, the values of total stresses of the underground pipeline along the coordinate are of a different nature and have certain small bursts due to the joints flexibility.

The results show that at each joint, as in the case in Figure 5 , the values of total stresses in underground pipeline along the coordinate have different pattern with certain small burst.

Figure 6 shows the changes in total stresses $\left(\sigma_{\mathrm{z}}^{+}, \sigma_{\mathrm{z}}{ }^{-}\right)$along the underground pipeline axis at a fixed time.

From the graphs in Figures 5 and 6, it is seen that each section of the underground pipeline affects the values of bending moments.

It can be seen from the results, the values of bending moment of the underground pipeline are separately formed at each section of the pipeline and at the joints.

At the joints, the values of bending moments are much smaller, compared with their values in sections, due to the flexibility of joints. The burst in value of bending moment near the wave front is associated with the pipeline end embedding in the deflection points. 


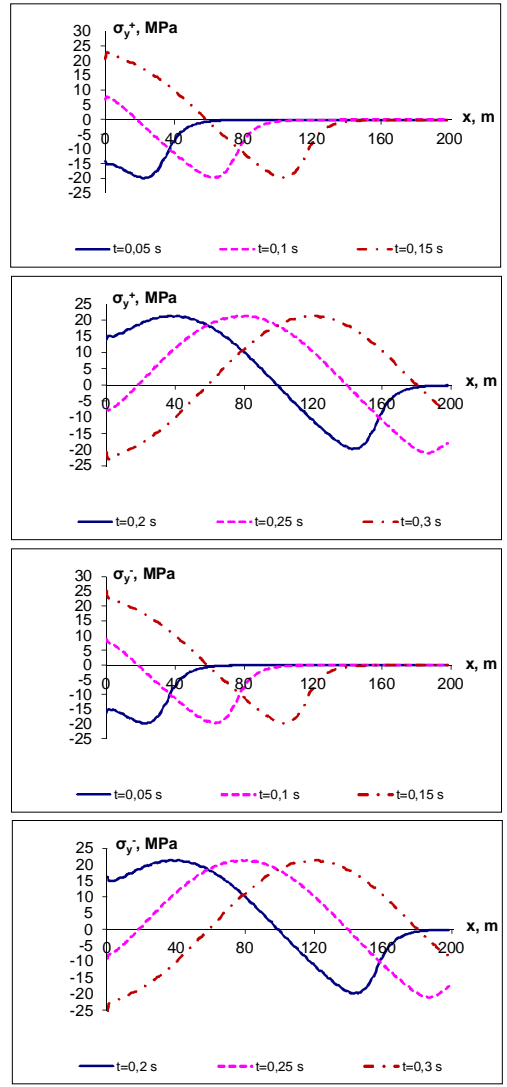

Figure 5: Changes in total stresses $\left(\sigma_{\mathrm{y}}{ }^{+}, \sigma_{\mathrm{y}}{ }^{-}\right)$along the underground pipeline axis at a fixed time

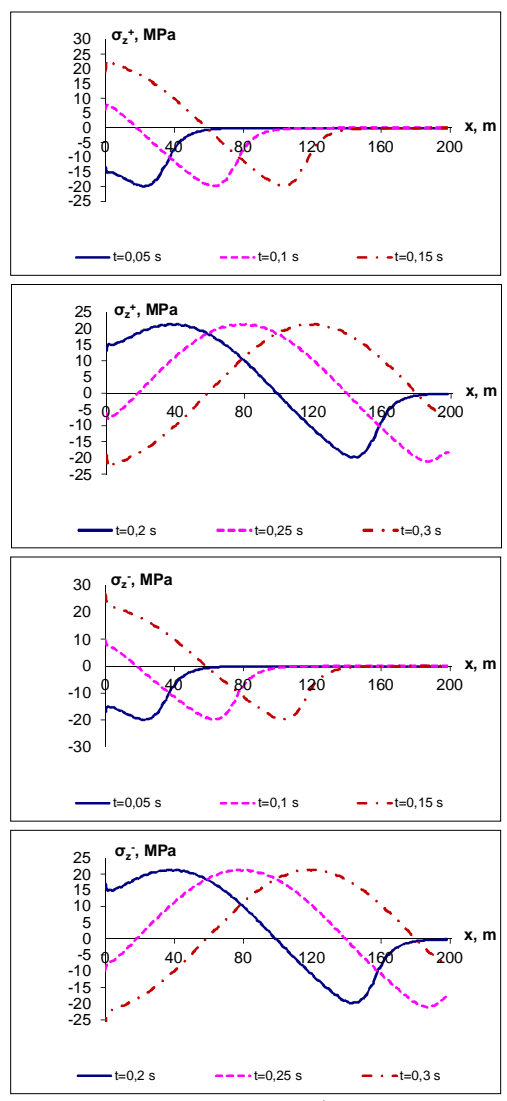

Figure 6: Changes in total stresses $\left(\sigma_{\mathrm{z}}^{+}, \sigma_{\mathrm{z}}{ }^{-}\right)$along the underground pipeline axis at a fixed time
For visualization, Figure 7 shows the change in bending moments values $\left(M_{y}, M_{z}\right)$ for a different range of lengths $(\mathrm{x}=5 \div 25 \mathrm{~m}$ and $\mathrm{x}=170 \div 195 \mathrm{~m})$ of the underground pipeline at a fixed time.

The figures show that the bending moments $\left(M_{y}, M_{z}\right)$ change along the length of the underground pipeline section; they increase from the left joint and then decrease to the right joint (see Figure 7), and at the joint the values of bending moment are less than in the middle of the section.

The previous graphs show the flexibility effect of section joints of the pipeline on the forces and moments distribution along the pipeline.

From the results obtained for the bending moment, it is seen that a separate bending moment arises in each section of the joined underground pipeline during earthquakes. Therefore, the dynamic process in an underground pipeline is of a complex pattern and the values of total stress increase. A seismic wave acts on a joined pipeline; calculations should be carried out at an angle to its longitudinal axis using a composite model of the pipeline, where each section of a pipeline and each of its joints are taken into account.

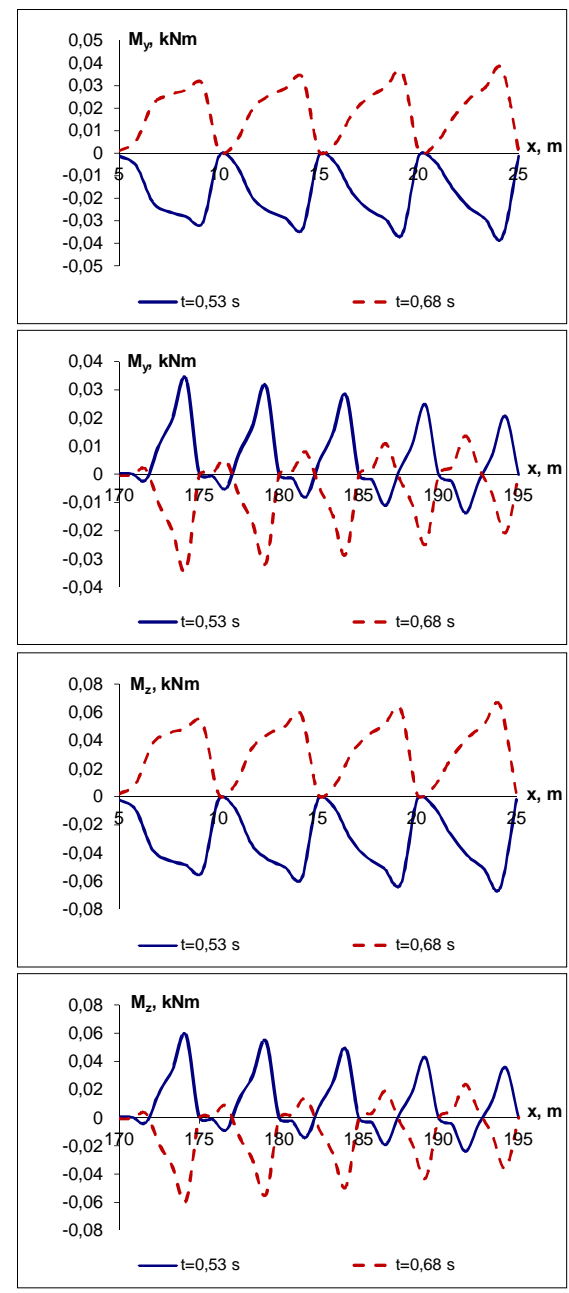

Figure 7: Change in bending moment (bending relative to axes $O y$ and $O z$ ) in different sections of the underground pipeline at a fixed time 


\section{CONCLUSION}

A generalized system of differential equations of underground pipeline spatial vibrations under random seismic actions at natural initial conditions based on the Hamilton-Ostrogradsky variational principle was developed. The system of differential equations with corresponding boundary and initial conditions is derived.

As seen from results of numerical experiments, based on hypothesis taken in calculations concerning the forces of interaction between long underground structures (pipelines) and soil, a good agreement is stated with actual data obtained from analysis of earthquake aftermath.

Joints of the underground pipeline play an important role in the stress-strain state formation, since the total stresses change in each section of underground pipeline due to the bending strains in the sections.

The problems were solved by the Finite difference method with consideration of combined longitudinal and transverse equations of oscillations of the system of pipelines, various mechanical, geometrical parameters and the modes of seismic loads. Developed mathematical models, algorithms and a complex of applied programs for the study of combined longitudinal and transverse oscillations of underground pipelines are described in details at arbitrary direction of seismic loading. So, new results were obtained for the systems of life support in seismodynamics of underground pipelines.

\section{REFERENCES}

1. T.R.Rashidov and D.A.Bekmirzaev, "Seismodynamics of Pipelines Interacting with Soil", Soil Mechanics and Foundation Engineering, Issue 3, Vol. 52, No. 3, pp. 149-154, 2015.

2. T.R. Rashidov, T. Yuldashev and D.A. Bekmirzaev, "Seismodynamics of underground pipelines with arbitrary direction of seismic loading", Soil Mechanics and Foundation Engineering, Issue 4, Vol. 55, No. 4, pp. 243-247, 2018.

3. D.A. Bekmirzaev, I. Mirzaev, "Dynamic processes in underground pipelines of complex orthogonal configuration at different incidence angles of seismic effect", International Journal of Scientific \& Technology Research, Vol. 9, No. 4, pp. 2449-2453, 2020.

4. S. Sarioletlagh, M. Nekooei, A. V. Oskouei, and A. Aziminejad, "Experimental and numerical modeling of horizontally-bent buried pipelines crossing fault slip", Latin American Journal of Solids and Structures, Vol. 16, No. 3, pp. 1-16, 2019.

5. D. Ha, T. H. Abdoun, M. J. O'Rourke, M. D. Symans, "Centrifuge Modeling of Earthquake Effects on Buried High-Density Polyethylene (HDPE) Pipelines Crossing Fault Zones", Journal of Geotechnical and Geoenvironmental Engineering, Vol. 134, No. 10, pp. 1501-1515, 2008.

6. M. Saberi, H. Arabzadeh, A. Keshavarz, "Numerical Analysis of Buried Pipelines with Right Angle Elbow under Wave Propagation", Procedia Engineering, Vol. 14, pp. 3260-3267, 2011.

7. P. Vazouras, S. A. Karamanos, P. Dakoulas, "Finite element analysis of buried steel pipelines under strike-slip fault displacements", Soil Dynamics and Earthquake Engineering, Vol. 30, pp. 1361-1376, 2010.

8. S. C. Chian, S. P. G. Madabhushi, "Effect of buried depth and diameter on uplift of underground structures in liquefied soils", Soil Dynamics and Earthquake Engineering, Vol. 41, pp. 181-190, 2012.

9. N.A. Nishonov, D.A. Bekmirzaev, E.V. An, Z. Urazmukhamedova, and K. Turajonov, "Behaviour and Calculation of Polymer Pipelines Under Real Earthquake Records", IOP Conference Series: Materials Science and Engineering, Vol. 869, 052076, 2020. doi:10.1088/1757-899X/869/5/052076.

10. D.A. Bekmirzaev, N.Sh. Mansurova, N.A. Nishonov, E.A. Kosimov, A.T. Numonov, "Solution of the problem of dynamics of underground pipelines under longitudinal seismic loading", IOP Conference Series: Materials Science and Engineering, Vol. 883, 012045, 2020. doi:10.1088/1757-899X/883/1/012045.

11. D.A. Bekmirzaev, I. Mirzaev, N.Sh. Mansurova, E.A. Kosimov, D.P. Juraev, "Numerical methods in the study of seismic dynamics of underground pipelines", IOP Conference Series: Materials Science and Engineering, Vol. 869, 052035, 2020. doi:10.1088/1757-899X/869/5/052035.

12. O.T. Cherney, Z.V.Smirnova, O.I. Vaganova, V.A. Razorenov, "Strengthening Technologies of Different Types of Foundations in Buildings", International Journal of Emerging Trends in Engineering Research, Vol. 8. No. 6, pp 2338-2342, 2020.

13. M. Hitesh, P. Subarna, K.R. Amiya, S.A. Edalatpanah, K. Ranjan, "A Tutorial on PowerShell Pipeline and its Loopholes", International Journal of Emerging Trends in Engineering Research, Vol. 8. No. 4, pp 975-982, 2020.

14. V. Corrado, B. D'Acunto, N. Fontana and M. Giugni, "Inertial Effects on Finite Length Pipe Seismic Response", Mathematical Problems in Engineering, Vol. 2012, pp 1-14, 2012. 